\title{
Inhibition of carbonic anhydrase IX (CA9) sensitizes renal cell carcinoma to ionizing radiation
}

\author{
WILHELMINA C.M. DUIVENVOORDEN ${ }^{1,2}$, SARAH N. HOPMANS ${ }^{1}$, \\ DANIEL GALLINO $^{3}$, THOMAS FARRELL ${ }^{3,4}$, CARRIE GERDES $^{4}$, DIANA GLENNIE ${ }^{3}$, \\ HIMU LUKKA $^{4,5}$ and JEHONATHAN H. PINTHUS ${ }^{1,2}$ \\ ${ }^{1}$ Department of Surgery, Division of Urology, McMaster University, Hamilton; ${ }^{2}$ Research Institute of St. Joe's,
St. Joseph's Healthcare, Hamilton; ${ }^{3}$ Department of Medical Physics, McMaster University, Hamilton;
${ }^{4}$ Juravinski Cancer Centre, Hamilton; ${ }^{5}$ Department of Oncology, McMaster University, Hamilton, ON, Canada
}

Received February 27, 2015; Accepted March 30, 2015

DOI: $10.3892 /$ or.2015.4184

\begin{abstract}
While normal kidneys are relatively sensitive to ionizing radiation (IR), renal cell carcinoma (RCC) is considered radioresistant. Carbonic anhydrase IX (CA9), an enzyme that maintains intracellular $\mathrm{pH}$ by carbon dioxide dissolution, is upregulated in the majority of RCC, but not in normal kidneys. Since regulation of intracellular $\mathrm{pH}$ may enhance radiation effects, we hypothesized that inhibition of CA9 may radiosensitize RCC. Clonogenic survival assay of human clear cell RCC 786-O and murine RCC RAG cells in the presence of a pharmacological CA9 inhibitor or with shRNA-mediated knockdown of CA9 was performed to investigate the response to IR in vitro (single dose or fractionated) and in vivo. Extracellular $\mathrm{pH}$ changes were measured in vitro. Treatment with AEBS [4-(2-aminoethyl)benzene sulfonamide], a sulfonamide, was used as a pharmacological inhibitor of the enzymatic activity of CA9. Nude mice bearing subcutaneous xenografts of 786-O cells stably expressing CA9 shRNA or scrambled control were irradiated (6 Gy). Tumor growth was followed longitudinally in the 786-O-bearing mice receiving AEBS (50-200 $\mu \mathrm{g} / \mathrm{ml}$ drinking water) or control (vehicle only) which were irradiated (6 Gy) and compared with mice receiving either IR or AEBS alone. In vitro inhibition of CA9 activity or expression significantly sensitized RCC cells to the effects of IR $(\mathrm{p}<0.05)$, an effect even more significant when hypofractionated IR was applied. In vivo irradiated xenografts from RCC cells transfected with CA9 shRNA were significantly smaller compared to irradiated xenografts from
\end{abstract}

Correspondence to: Dr Jehonathan H. Pinthus, Juravinski Hospital, Surgical Oncology, 3rd floor B148, 711 Concession Street, Hamilton, ON L8V 1C3, Canada

E-mail: jehonathan.pinthus@jcc.hhsc.ca

Abbreviations: AEBS, 4-(2-aminoethyl)benzene sulfonamide; CA9, carbonic anhydrase IX; ccRCC, clear cell renal cell carcinoma; IR, ionizing radiation; RCC, renal cell carcinoma

Key words: carbonic anhydrase IX, radiation therapy, renal cell carcinoma, sulfonamide the scrambled shRNA controls $(\mathrm{p}<0.05)$. RCC xenografts from mice treated with AEBS in combination with IR grew significantly slower than all controls $(p<0.05)$. Inhibition of CA9 expression or activity resulted in radiation sensitization of RCC in a preclinical mouse model.

\section{Introduction}

Partial or radical nephrectomy for primary renal cell carcinoma (RCC) achieves excellent rates of cure (1), yet the procedure is invasive and often results in loss of normal renal parenchyma leading to the development of renal insufficiency with its associated long-term morbidity and even mortality (1). Radiation treatment of primary RCC is rarely employed for curative intent as RCC is generally believed to be radiationresistant. Of note, in clinical practice, particular care is taken to keep kidney radiation doses within acceptable tolerance limits as normal renal parenchyma is considered relatively sensitive to radiation (2). One possible explanation for this conundrum may be the differential expression of proteins, such as carbonic anhydrase IX (CA9), involved in radiation resistance in RCC cells compared to normal renal cells. CA9 is not expressed in normal kidney cells (3), yet its expression is ubiquitous in clear cell RCC (ccRCC), most likely due to the fact that expression of CA9 is transcriptionally regulated by hypoxia-inducible factor-1 $\alpha$ (4), which accumulates in ccRCC cells as a result of frequent inactivating mutations in the von Hippel-Lindau tumor-suppressor gene (5).

The family of carbonic anhydrase enzymes catalyzes the dissolution of $\mathrm{CO}_{2}$ in water as carbonic acid and protons (6). CA9 contributes to the acidification of the local tumor environment and guards tumor cells against acidosis. We hypothesized that upregulation of CA9 in RCC cells may account, at least in part, for the radiation resistance of RCC, and thus targeting CA9 expression or enzymatic activity may sensitize RCC to ionizing radiation.

\section{Materials and methods}

Cell culture and transfection. Mycoplasma-free human ccRCC 786-O and human prostate adenocarcinoma LNCaP 
cells (ATCC, Manassas, VA, USA) were propagated in RPMI1640 medium supplemented with 10\% FBS (Invitrogen, Burlington ON, Canada). The 786-O cell identity was verified by STR analysis (ATCC). Murine RCC RAG cells and human glioblastoma LN-18 cells (ATCC) were maintained in Eagle's MEM and Dulbecco's MEM, respectively, supplemented with $10 \%$ FBS.

The shRNA vector for human CA9 (CA9 shRNA) and the non-effective negative scrambled control were purchased from Origene (Rockville, MD, USA). Transfection of human ccRCC 786-O cells was performed using 12\% Fugene (Promega, Madison, WI, USA). Cells stably transfected with shCA9 or the scrambled control were selected with $1.0 \mu \mathrm{g} / \mathrm{ml}$ puromycin (Sigma-Aldrich, Oakville, ON, Canada).

Clonogenic survival experiments. RAG, 786-O, and LN-18 cells (250 per well) were seeded onto 6-well plates in $3 \mathrm{ml}$ of medium and allowed to adhere by incubation at $37^{\circ} \mathrm{C}$ and $5 \%$ $\mathrm{CO}_{2}$ for $4 \mathrm{~h}$. For LNCaP, 1000 cells were seeded per well and allowed to adhere for $24 \mathrm{~h}$.

For the AEBS treatment, a stock solution of 4-(2-aminoethyl)benzene sulfonamide (AEBS, $33 \mathrm{mM}$, Sigma-Aldrich) was prepared fresh with $\mathrm{H}_{2} \mathrm{O}$ and filter-sterilized using a $0.2 \mu \mathrm{m}$ syringe filter. AEBS at concentrations ranging from $3.3 \mu \mathrm{M}$ to $3300 \mu \mathrm{M}$ was added to $100 \mu \mathrm{l}$ of $\mathrm{H}_{2} \mathrm{O}$ to $3 \mathrm{ml}$ media. Solvent controls were also included. The medium was aspirated and replaced with $3 \mathrm{ml}$ of the appropriate drug solutions in media in duplicate wells. After $24 \mathrm{~h}$ of incubation, the media were aspirated, and $3 \mathrm{ml}$ of fresh medium was added to each well.

Ionizing radiation (IR) of cells was performed using a Varian Linear Accelerator (LINAC) generating six MV X-rays (Varian Medical Systems, Inc., Palo Alto, CA, USA). Cells plated in duplicate 6-well plates were irradiated at a distance of $100 \mathrm{~cm}$ in a $16 \mathrm{~cm}$ by $20 \mathrm{~cm}$ field. A $19-\mathrm{mm}$-thick acrylamide sheet was placed on the plates as a build-up region. Thermoluminescent dosimeters were used to measure and calibrate the dose. The cells received from 1 to 8 Gy of $6 \mathrm{MV}$ $\mathrm{X}$-ray radiation.

Following treatment, the cells were incubated for an additional 6 days (786-O), 7 days (RAG), or 12 days (LNCaP and LN-18), after which the medium was aspirated and the colonies were stained with crystal violet $(0.25 \%$ in $95 \%$ ethanol) for $10 \mathrm{~min}$. Colonies of 50 cells or more were counted. Survival was expressed as a percentage of the corresponding untreated controls, and $\mathrm{IC}_{50}$ values were calculated using CalcuSyn software version 1.2 (Biosoft, Cambridge, UK). Experiments were repeated three times.

CA9 activity assay. Confluent RCC cells in 6-well plates were washed twice with $3 \mathrm{ml}$ of PBS. One $\mathrm{ml}$ of $0.9 \%$ saline (adjusted to $\mathrm{pH} 8.0$ with $\mathrm{NaOH}$ ) containing $0.15 \mathrm{mg} / \mathrm{ml}$ phenol red was added to each well. Deionized water $(100 \mu \mathrm{l})$ with or without AEBS at various concentrations was added to the wells. Starting $5 \mathrm{sec}$ after the addition of saline to the cells, the absorbance at $565 \mathrm{~nm}$ was measured using a Powerwave HT spectrophotometer (BioTek, Winooski, VT, USA) at $1 \mathrm{sec}$ intervals for $20 \mathrm{sec}$. The relative absorbance of a particular well throughout the $20 \mathrm{sec}$ was determined as a percent of the no cell control average at that time interval ( $\left.\mathrm{A} / \mathrm{A}_{\text {no cells }}\right)$.
Western blot analysis. Cultured cells were lysed using RIPA lysis buffer. Lysate $(40 \mu \mathrm{g})$ was resolved on a $10 \%$ SDS-PAGE gel and transferred onto nitrocellulose membrane. Primary antibodies used were CA9 (1:1,000 Epitomics, Burlingame, CA, USA) and $\beta$-actin (1:2,000, Sigma-Aldrich). Secondary HRP-conjugated antibody (1:200, Dako, Carpinteria, CA, USA) was used in conjunction with chemiluminescence detection.

Animal studies. All protocols for animal studies were reviewed and approved by the institutional Animal Research Ethics Board (AUP\# 12-09-37). Per group, 7-10 female inbred nude (Balb/c nu/nu) mice (Charles River, St. Constant, QC, Canada) 5 weeks of age were used. 786-O parental, shCA9 or scram-

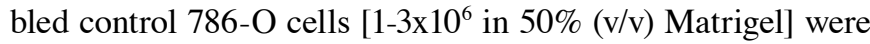
injected subcutaneously into the right flank of each mouse. Tumor size was measured every three days using Vernier calipers, and the tumor volume was determined using the formula $\pi / 6$ (length $\mathrm{x}$ width $\mathrm{x}$ height) until the largest tumor reached $400 \mathrm{~mm}^{3}$. The mice were sacrificed 7-12 weeks after tumor cell injection, when the tumors were dissected, weighed, fixed in formalin and embedded in paraffin.

For mice in the IR group, 21-27 days post injection, when tumors were palpable, the animals were anaesthetized using isoflurane and positioned in sterile, acrylamide cylinders connected to a portable anaesthetic machine. Cylinders were transported to the treatment area, where they were positioned at a distance of $100 \mathrm{~cm}$ to the source and irradiated with $6 \mathrm{~Gy}$ in a $2 \mathrm{~cm}$ by $2 \mathrm{~cm}$ field using a Varian Linear Accelerator generating $6 \mathrm{MV}$ X-rays. A 5-mm-thick sheet of superflab bolus material served as a build-up region. Animals in non-irradiated control groups were anaesthetized for a similar time period.

Animals in the AEBS-treatment groups received 50 or $200 \mu \mathrm{g} / \mathrm{ml}$ AEBS in the drinking water supplied fresh every two days starting two days before IR. No adverse effects of the treatment were observed.

The serum levels of VEGF were determined using a mouse VEGF ELISA kit (R\&D Systems, Minneapolis, MN, USA) according to the manufacturer's instructions on a BMG Labtech SpectroStar Nano multi-well plate reader. Immunostaining of $4 \mu \mathrm{m}$-thick tumor xenograft sections for CD31 and subsequent image analysis was performed as previously described (7) resulting in the microvessel density expressed as endothelial length (in $\mu \mathrm{m}$ ) per $\mathrm{mm}^{2}$.

AEBS mass spectrometry. Trifluoroacetic acid $(2 \mu \mathrm{l})$ and methanol $(500 \mu \mathrm{l})$ were added to $100 \mu \mathrm{l}$ mouse serum, vortexed for $10 \mathrm{sec}$ and centrifuged at $5000 \mathrm{rpm}$ for $10 \mathrm{~min}$. Resulting supernatant (300 $\mu \mathrm{l})$ was removed and blown down to dryness with nitrogen. The sample was reconstituted in $200 \mu 1$ of methanol/water (1:1) containing $25 \mu \mathrm{g} / \mathrm{ml}$ phenylalanine (internal standard) and filtered using a $13-\mathrm{mm}$ syringe filter ( $0.2 \mu \mathrm{m}$ GHP membrane). The sample $(5 \mu \mathrm{l})$ was run on the LC-MS (Agilent 6340 Ion Trap coupled to an Agilent 1200 HPLC, Agilent Technologies Inc, Mississauga, ON, Canada) at the McMaster regional centre for mass spectrometry. Analysis was performed using multiple reaction monitoring on AEBS and phenylalanine with the transition at 201-184 (m/z) and 166-120 (m/z), respectively. Control serum was spiked with AEBS at $1-16 \mu \mathrm{g} / \mathrm{ml}$. 
A

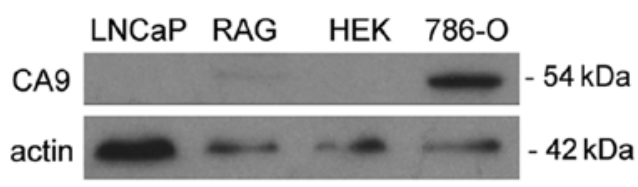

B

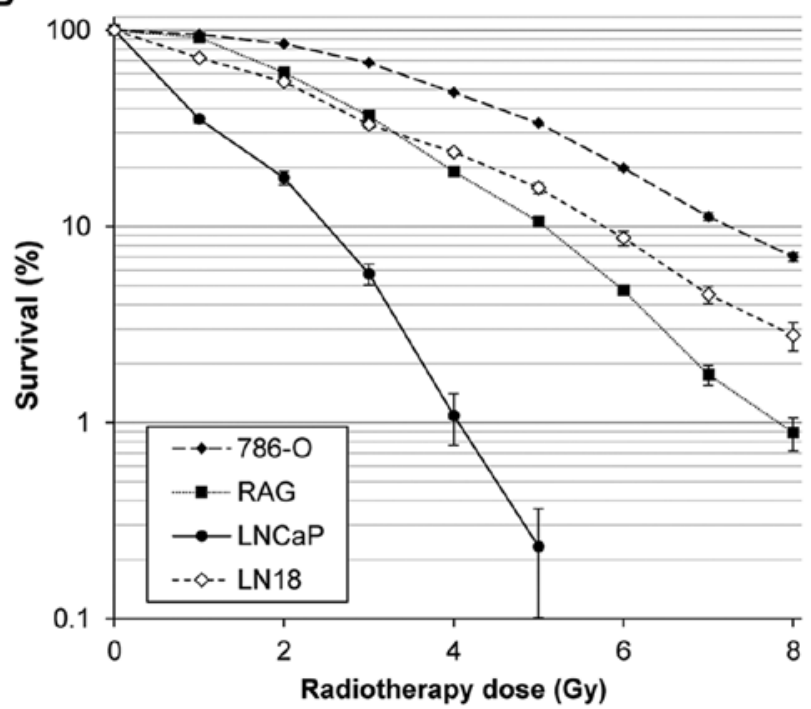

Figure 1. CA9 expression and clonogenic survival after ionizing radiation (IR). (A) CA9 protein expression was detected in human ccRCC 786-O and murine RCC RAG cells, but not in human embryonic kidney HEK293 or human prostate adenocarcinoma LNCaP cells. CA9 was detected by western blot analysis. The protein expression of $\beta$-actin served as a loading control. (B) Clonogenic survival after IR as measured by colony forming assay. Values are expressed as a percentage of the corresponding control. Each value represents mean data \pm standard error of the mean (SEM) of three experiments carried out in duplicate.

Statistical analysis. Values are expressed as the mean \pm the standard error of the mean. Where appropriate, results are presented with $95 \%$ confidence intervals (CI). Dependent on whether the data were normally distributed or not, parametric (Student's t-test) or nonparametric methods (Mann-Whitney U-test) were used with a p-value $<0.05$ indicative of statistical significance.

\section{Results}

CA9 is present in the radiation-resistant 786-O and $R A G$ $R C C$ cells. Protein expression of CA9 was determined in the lysates of human prostate adenocarcinoma LNCaP, human ccRCC 786-O, murine RCC RAG and immortalized human embryonic kidney HEK-293 cells by western blot analysis (Fig. 1A). CA9 was detectable in the 786-O and RAG cells, but not in the LNCaP or HEK-293 cells. Clonogenic survival experiments demonstrated that RAG cells were more sensitive to IR than 786-O cells $(\mathrm{p}<0.05)$, whereas LNCaP cells were the most sensitive among the cell lines tested (Fig. 1B). Human glioblastoma LN-18 cells exhibited similar tolerance to IR as the RAG cells. The 786-O cells displayed significantly decreased survival at a dose of 2 Gy and above $(\mathrm{p}<0.001)$, whereas LNCaP cells displayed significantly decreased survival at all radiation doses $(\mathrm{p}<0.001)$. The calculated $\mathrm{IC}_{50}$ values for each cell line are presented in Table I.

Knockdown of CA9 expression by shRNA in 786-O cells leads to radiosensitivity. To investigate the significance of CA9 expression on RCC radiosensitivity, human ccRCC 786-O subclones stably expressing shRNA specific for CA9 were generated. These shCA9 cells showed 92\% knockdown of CA9 protein expression compared to the respective control cells (scrambled shRNA) (Fig. 2A). Clonogenic survival of the 786-O scrambled control and shCA9 cells was determined after IR with increasing doses of 1-8 Gy and clearly demonstrated
Table I. Sensitivity of the different cell lines to ionizing radiation in vitro as determined by the $\mathrm{IC}_{50}$ value measured by clonogenic survival.

\begin{tabular}{lcc}
\hline Cell line & $\mathrm{IC}_{50}(\mathrm{~Gy})$ & $\begin{array}{c}95 \% \text { confidence } \\
\text { interval }(\mathrm{Gy})\end{array}$ \\
\hline Human ccRCC 786-O & 3.52 & $3.22-3.85$ \\
$\begin{array}{l}\text { Murine RCC RAG } \\
\text { Human prostate }\end{array}$ & 2.29 & $2.05-2.56$ \\
$\begin{array}{l}\text { adenocarcinoma LNCaP } \\
\text { Human glioblastoma LN18 }\end{array}$ & 1.01 & $0.65-1.57$ \\
\hline
\end{tabular}

that knockdown of CA9 confers sensitivity to IR ( $\mathrm{p}<0.001)$. The $\mathrm{IC}_{50}$ value decreased by $>50 \%$, from $3.64 \mathrm{~Gy}(95 \% \mathrm{CI}$ : $3.27-4.05)$ in the scrambled control cells to $1.81 \mathrm{~Gy}(95 \% \mathrm{CI}$ : 1.44-2.27) in the shCA9 cells (Fig. 2B). To further demonstrate the effect of CA9 knockdown in vitro, the activity of the CA9 enzyme was measured using phenol red as an indicator. In comparison to the scrambled control cells, shCA9 cells had significantly decreased acidification capacity $(\mathrm{p}<0.001)$ and experienced $51 \%$ of the absorbance change observed in the scrambled control cells (Fig. 2C).

The effect of CA9 knockdown on the in vivo growth after subcutaneous injection of shCA9 or scrambled control cells $\left(1 \times 10^{6}\right)$ into nude mice ( $\mathrm{n}=7 /$ group) was determined. A tumor take rate of $64 \%$ was achieved. The tumor volume from mice injected with the shCA9 cells was not significantly different from mice injected with cells transfected with the scrambled control shRNA (Fig. 3A and B). However, when the subcutaneous tumors were also irradiated 21 days after cell injection, we observed that IR of the shCA9-transfected 786-O cells led to decreased tumor growth $(\mathrm{p}<0.001)$ and a $78.7 \%$ decrease 
A

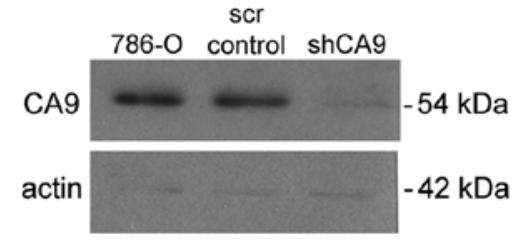

B 100

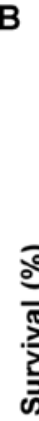

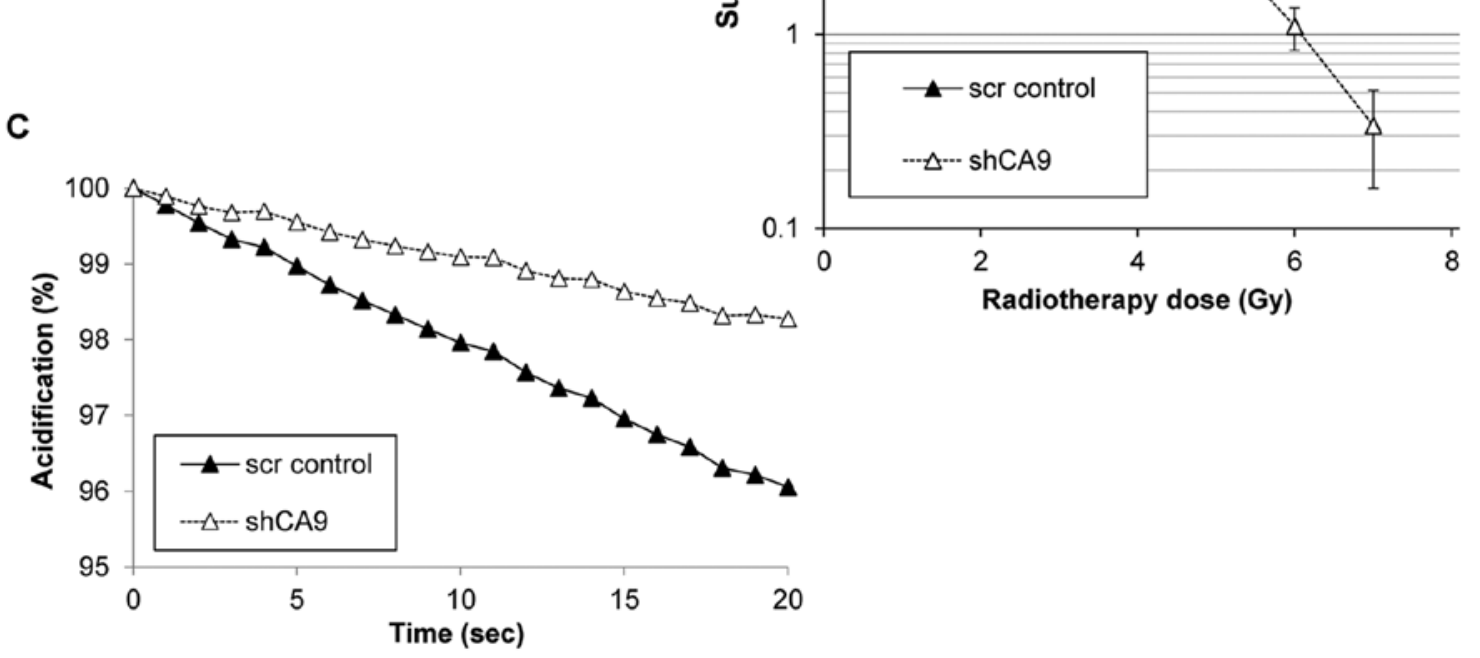

Figure 2. Effect of shRNA-mediated knockdown of CA9 on acidification and clonogenic survival after ionizing radiation (IR). (A) Subclones of human ccRCC 786-O cells demonstrated a 92\% knockdown of CA9 protein expression in cells stably expressing shRNA specific for CA9 (shCA9) compared to corresponding scrambled control-transfected cells (scr control). CA9 was detected by western blot analysis. The protein expression of $\beta$-actin served as a loading control. (B) Clonogenic survival of human ccRCC 786-O cells expressing shCA9 or scrambled control shRNA after IR as measured by colony forming assay. Values are expressed as a percentage of the corresponding control. Each value represents mean data ( \pm SEM) of three experiments carried out in duplicate. (C) Acidification of the medium from 786-O cells expressing shCA9 or scrambled control shRNA as determined by the decrease in absorbance of phenol red over time. Decrease in absorbance is expressed as relative absorbance at $565 \mathrm{~nm}$ at a given time point (in sec) over the absorbance at time zero. Background absorbance change was subtracted at each time interval. Error bars represent SEM for six replicates.

in tumor volume at sacrifice (Fig. 3B). IR of the scrambled control-injected mice led to a $20.7 \%$ reduction in tumor volume after 12 weeks (Fig. 3A). Western blot analysis of the tumor homogenates showed that CA9 remained reduced in the shCA9-injected mice (53\%) at sacrifice, 12 weeks after tumor cell injection (Fig. 3C).

AEBS inhibits CA9 activity in vitro and leads to radiosensitivity. AEBS is a known inhibitor of CA9's enzymatic reaction with a $\mathrm{Ki}$ of $33 \mathrm{nM}(8)$. However, to the best of our knowledge, AEBS has not been used previously to inhibit CA9 in vivo, in contrast to acetazolamide, a similar sulfonamide. In the presence of $33 \mu \mathrm{M}$ AEBS, the human ccRCC 786-O cells exhibited a significant decrease in clonogenic survival after IR when compared to the untreated control (Fig. 4A, p<0.001). Similarly, the more radiation-sensitive murine RAG cells also exhibited a decrease in clonogenic survival after IR when treated with the same concentration of AEBS for $24 \mathrm{~h}$ (Fig. 4B, $\mathrm{p}=0.018$ ). To further demonstrate the effectiveness of AEBS in vitro, the acidification of the extracellular environment of 786-O cells was determined (Fig. 4C). In comparison to the untreated control 786-O cells, incubation with either $33 \mu \mathrm{M}$ or $33 \mathrm{nM}$ AEBS caused significantly less acidification $(\mathrm{p}<0.001)$, leading to a decrease of 70 and $36 \%$, respectively. AEBS was not cytotoxic; by clonogenic survival the $\mathrm{IC}_{50}$ values for AEBS amounted to 1,360 and $>5,000 \mu \mathrm{M}$ in the $786-\mathrm{O}$ and $\mathrm{RAG}$ cells, respectively (data not shown).

Radiosensitization is more pronounced when the radiation is hypofractionated. To investigate whether a hypofractionated regimen of radiation delivery could increase the survival difference between CA9-inhibited and non-inhibited RCC cells, 786-O cells expressing scrambled control shRNA or shCA9 or with and without the addition of AEBS to the medium were subjected to either one treatment of 6 Gy or three treatments of $2 \mathrm{~Gy}$ for three consecutive days. Cells receiving AEBS or expressing shCA9 had significantly reduced survival $(\mathrm{p}<0.001)$ after receiving a single dose treatment of $6 \mathrm{~Gy}$ compared to the fractionated radiation treatment of three doses of 2 Gy (Fig. 5).

AEBS administration increases radiosensitivity in vivo. We determined the effect of CA9 inhibition on the in vivo growth after subcutaneous injection of 786-O cells $\left(3 \times 10^{6}\right)$ into nude mice ( $n=20 /$ group). A tumor take rate of $85 \%$ was achieved. Mice were treated with either AEBS starting 25 days after cell injections or received IR on day 27 and were sacrificed 24 days later. One untreated control group was included and one group of mice received both IR and AEBS. The tumor volume in mice treated with AEBS was significantly smaller than that in the control mice (Fig. 6A; $p=0.03$ ANOVA). When 
A

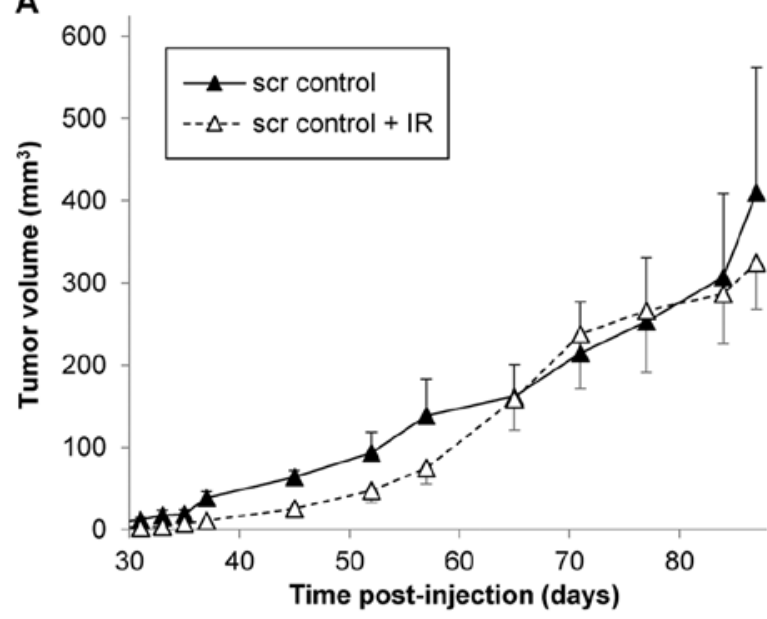

C
B

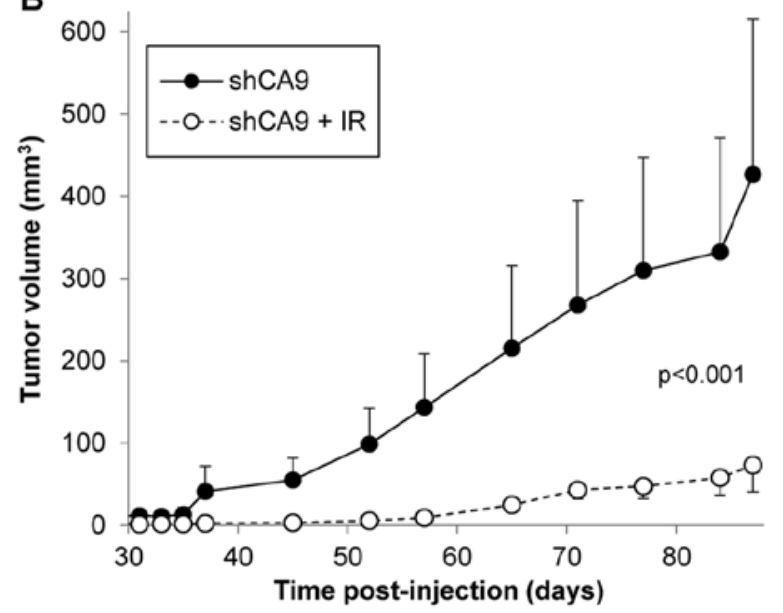

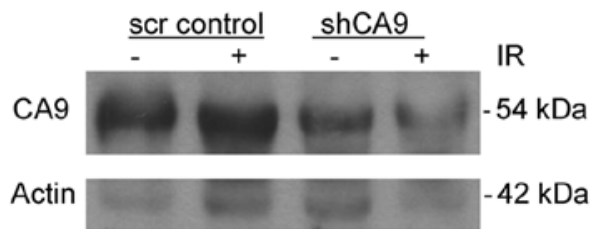

Figure 3. shRNA-mediated knockdown of CA9 sensitizes ccRCC xenografts to ionizing radiation (IR). (A) Longitudinal tumor growth of human ccRCC $786-\mathrm{O}$ cells in vivo. The tumor volume from mice injected with scrambled control-transfected 786-O cells $(\mathrm{n}=3)$ and treated with 6 Gy IR on day 21 was not significantly different from the tumor volume of the non-irradiated mice $(n=7)$. (B) The tumor volume from mice ( $\mathrm{n}=4$ /group) injected with shCA9 786-O cells and treated with 6 Gy IR on day 21 was significantly smaller than that from the non-irradiated mice ( $<<0.0001)$. (C) Tumors derived from human ccRCC 786-O cells demonstrated a 53\% knockdown of CA9 protein expression in cells stably expressing shRNA specific for CA9 (shCA9) compared to the corresponding scrambled control-transfected cells (scr control). CA9 was detected by western blot analysis. The protein expression of $\beta$-actin served as a loading control.

A

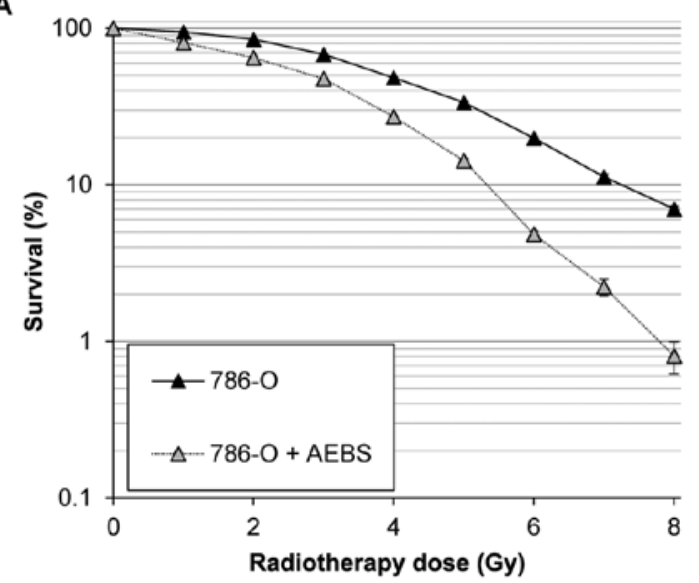

C

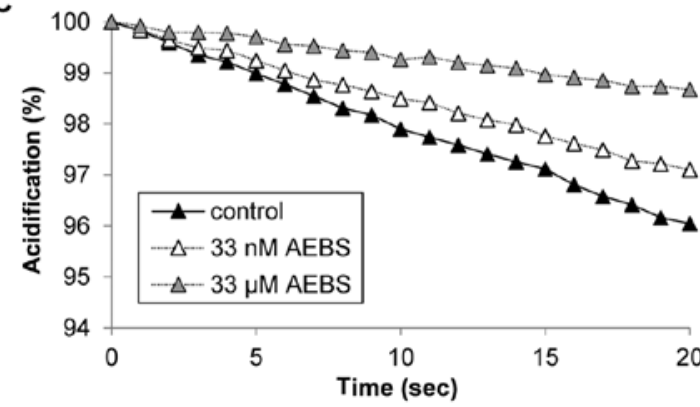

B

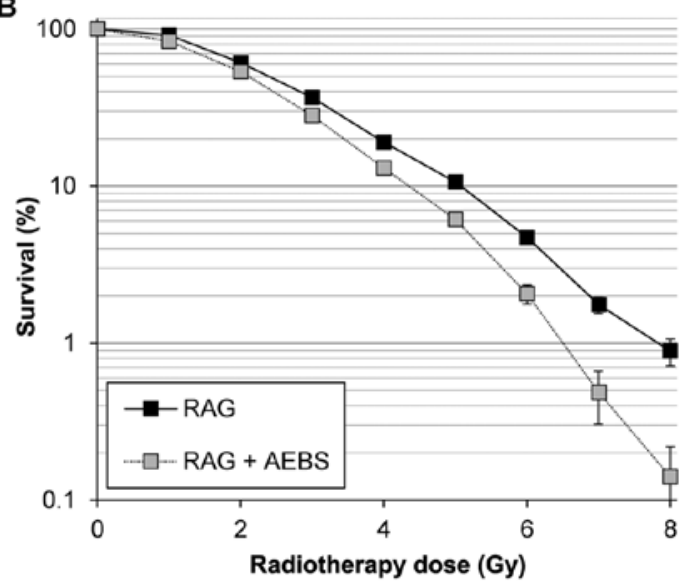

Figure 4. Effect of AEBS-mediated inhibition of CA9 on acidification and clonogenic survival after ionizing radiation (IR). (A) Clonogenic survival after IR of human ccRCC 786-O cells in the presence or absence of $33 \mu \mathrm{M}$ AEBS for $24 \mathrm{~h}$ as measured by colony forming assay. (B) Clonogenic survival after IR of murine RCC RAG cells in the presence or absence of $33 \mu \mathrm{M}$ AEBS for $24 \mathrm{~h}$ as measured by colony forming assay. Values are expressed as a percentage of the corresponding control. Each value represents mean data ( \pm SEM) of three experiments carried out in duplicate. (C) Acidification of the medium from the 786-O cells in the presence or absence of AEBS at $33 \mathrm{nM}$ or $33 \mu \mathrm{M}$ as determined by the decrease in absorbance of phenol red over time. Decrease in absorbance is expressed as relative absorbance at $565 \mathrm{~nm}$ at a given time point (in sec) over the absorbance at time zero. Background absorbance change was subtracted at each time interval. Error bars represent the SEM for six replicates. 
A

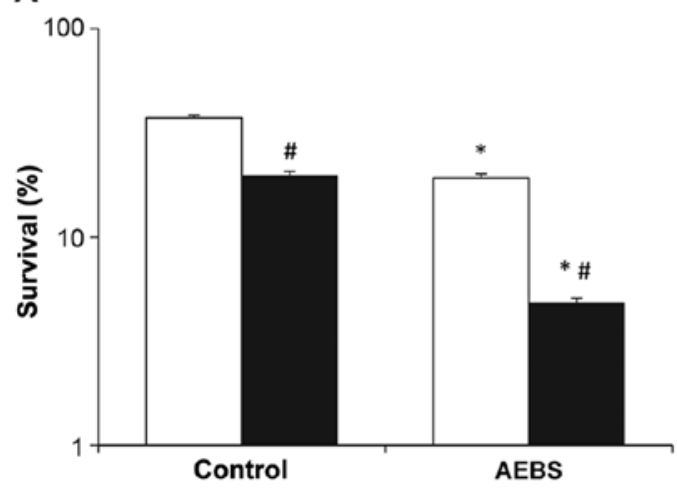

B

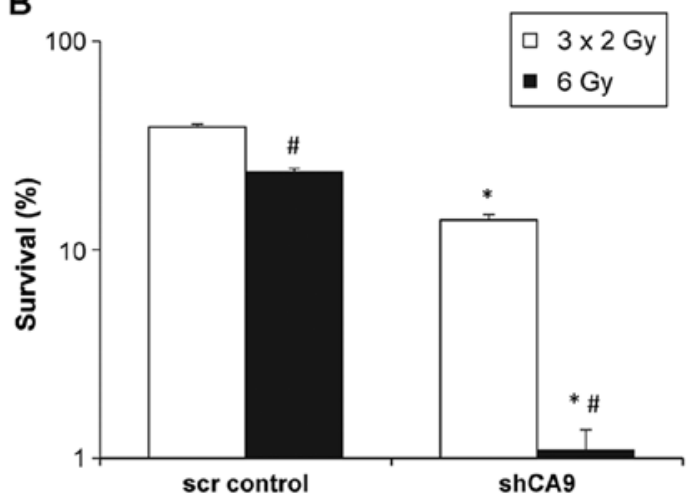

Figure 5. Effect of hypofractionation of the radiation dose on AEBS- and shCA9-mediated inhibition of CA9 on clonogenic survival. Radiation sensitivity of 786-O cells (A) in the presence or absence of $33 \mu \mathrm{M}$ AEBS and (B) expressing shRNA specific for CA9 as determined by clonogenic survival. Ionizing radiation (IR) consisted of a fractionated radiation regimen of three times $2 \mathrm{~Gy}$ radiotherapy doses compared with a single-dose (6 Gy). Fractions consisted of $2 \mathrm{~Gy}$ doses of six MV X-rays delivered once every $24 \mathrm{~h}$ for three days. Survival is expressed as a percentage, under each condition, of the untreated control. Error bars represent SEM of at least two experiments carried out in duplicate. ${ }^{*} \mathrm{p}<0.05$ vs. the corresponding control; ${ }^{*} \mathrm{p}<0.05 \mathrm{vs}$. the fractionated radiation dose.

A

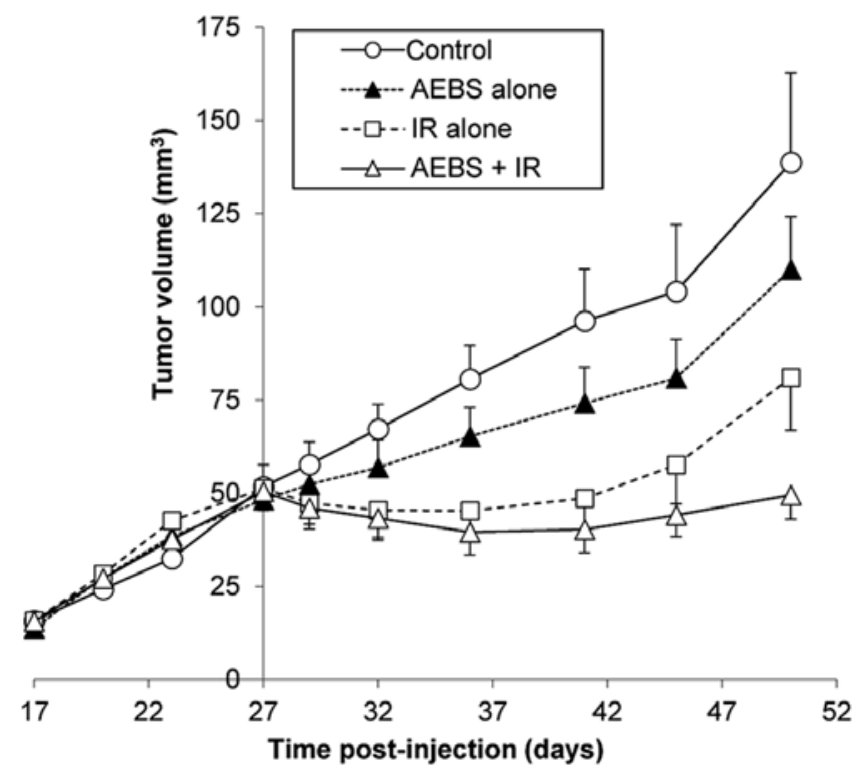

B

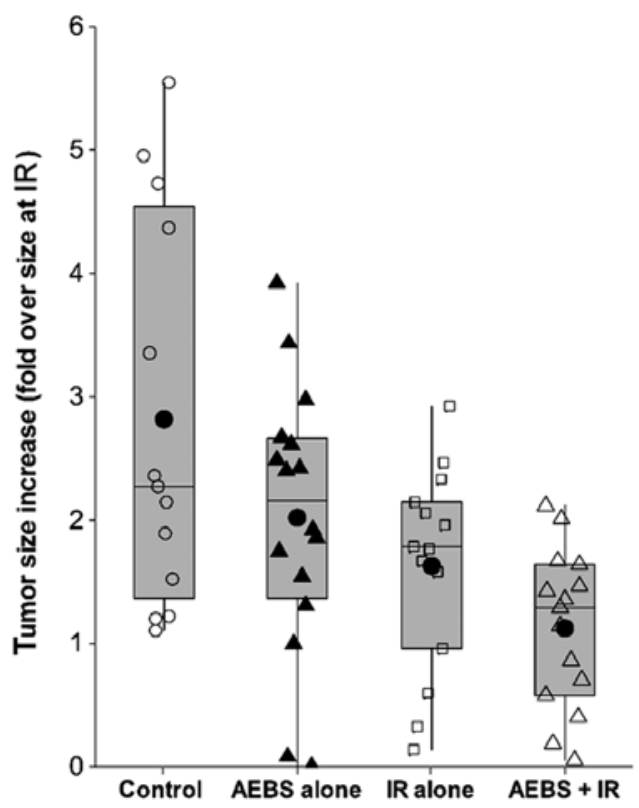

C

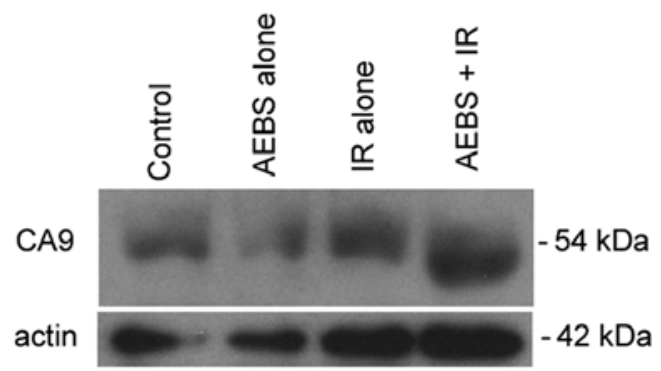

D

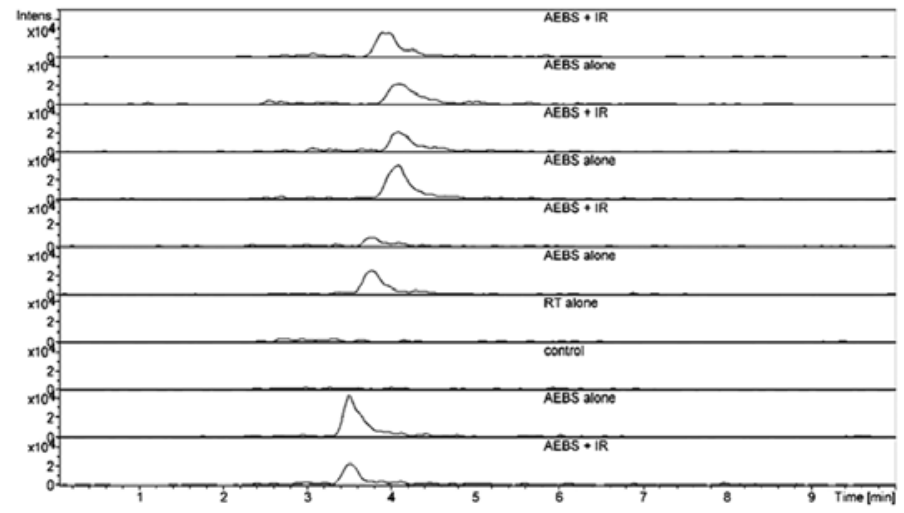

Figure 6. Inhibition of CA9 sensitizes ccRCC xenografts to ionizing radiation (IR). (A) Longitudinal tumor growth of human ccRCC 786-O cells in vivo. The tumor volumes from mice ( $\mathrm{n}=15$-16/group) after treatment with either 6 Gy IR on day 27 and/or AEBS starting on day 25 were all significantly smaller than the volume from the untreated control mice (ANOVA; $p<0.05$ ). The tumor volumes from mice treated with IR combined with AEBS were significantly smaller compared to the irradiated mice $(\mathrm{p}=0.04)$ and compared to mice treated with AEBS alone $(\mathrm{p}<0.0005)$. (B) Increase in tumor volume at endpoint expressed as fold increase over the tumor size at the time of IR (day 27). (C) Tumors derived from human ccRCC 786-O cells demonstrated comparable CA9 protein expression irrespective of the treatment of the mice. CA9 was detected by western blot analysis, and $\beta$-actin served as a loading control. (D) Examples of ion chromatograms to determine the concentration of AEBS in the mouse serum samples. Serum samples were obtained 51 days after subcutaneous injection of ccRCC $786-O$ cells and 26 days after start of the AEBS treatment. 
A

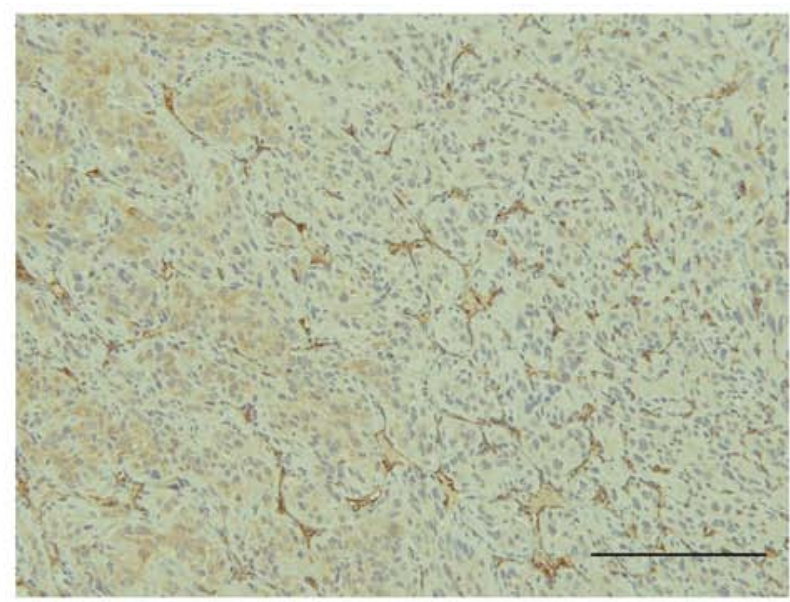

B

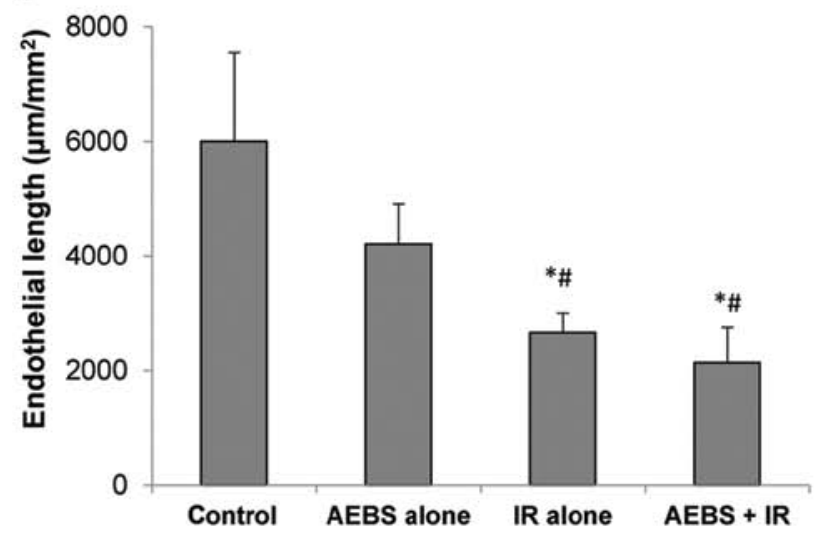

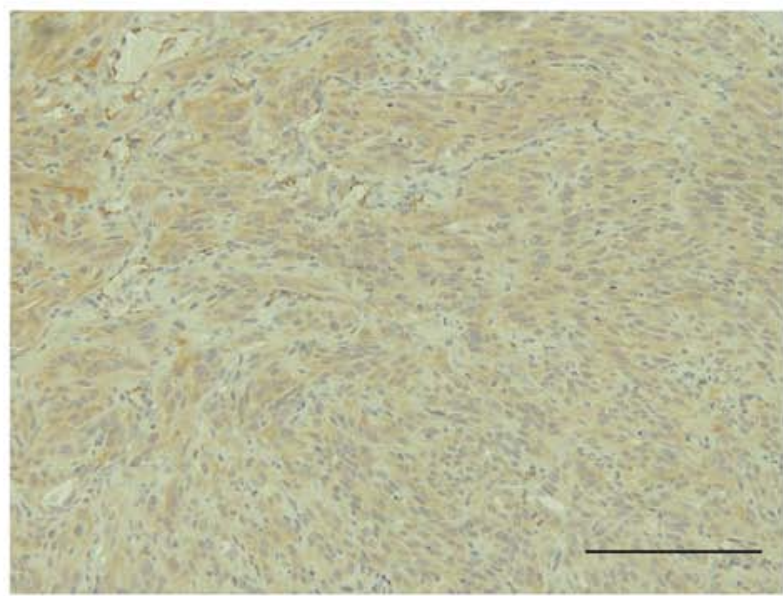

C

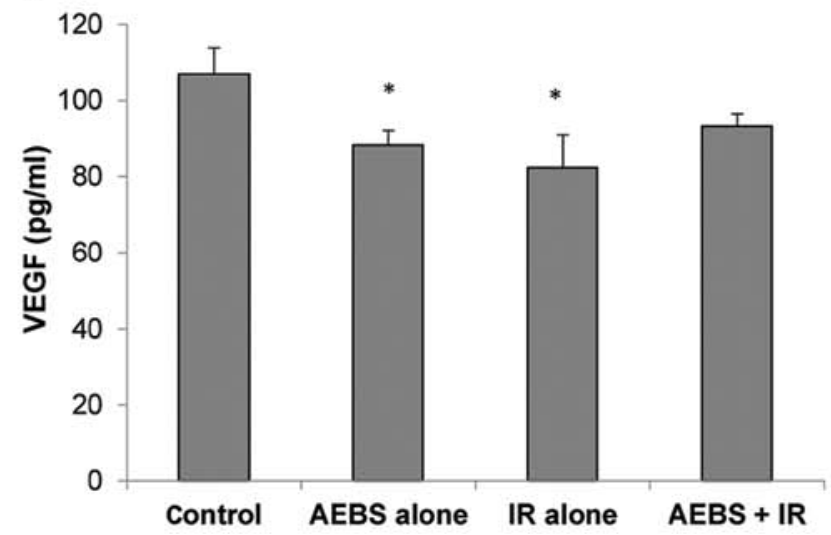

Figure 7. Microvessel density and serum VEGF levels in the ccRCC xenografts subjected to AEBS treatment and/or ionizing radiation (IR). (A) Microvessel staining by CD31-immunohistochemistry performed on tumor sections obtained 51 days after subcutaneous injection of ccRCC 786-O cells from the control mice or mice treated with AEBS and IR. The left panel shows staining from an untreated control tumor at $\mathrm{x} 200 \mathrm{magnification}$ (scale bar=200 $\mu \mathrm{m}$ ). The right panel shows staining of a tumor after combined treatment. (B) Linear endothelial length determined in the subcutaneous tumors of ccRCC 786-O cellinjected mice sacrificed 51 days after injection treated with either $200 \mu \mathrm{g} / \mathrm{ml}$ AEBS alone $(\mathrm{n}=6)$, IR $(\mathrm{n}=8)$, both ( $\mathrm{n}=7$ ) or control $(\mathrm{n}=6)$. Data shown represent mean \pm SEM. ${ }^{*}$ p $<0.05$ vs. control; ${ }^{*}$ p $<0.05$ vs. AEBS alone. (C) Serum VEGF levels at sacrifice (51 days after injection) of mice treated with either AEBS alone $(n=7)$, IR $(n=5)$, both $(n=6)$ or control $(n=8)$. Data represent mean \pm SEM. ${ }^{*} p<0.05$ vs. control.

the subcutaneous tumors were also irradiated 27 days after cell injection, we observed that the combination treatment led to decreased tumor grow th compared to either AEBS or IR alone ( $\mathrm{p}<0.0005$ and $\mathrm{p}=0.04$, respectively). At sacrifice, this led to an average increase of a mere $12 \%$ in tumor volume from the time of IR on day 27 to sacrifice on day 51 in the combination group (Fig. 6B). In comparison, the tumor volumes of the untreated control mice had increased in size by almost 3 -fold. Protein levels of CA9 in the mouse tumors did not differ between treatment groups (Fig. 6C). Mass spectrometry showed that measurable and CA9-inhibitory levels of AEBS were achieved in mouse serum (Fig. 6D). Levels reached $26.5 \mu \mathrm{M} 18 \mathrm{~h}$ after starting the treatment and remained similar throughout the experiment (data not shown).

Using immunohistochemical staining of the subcutaneous tumors for CD31, an endothelial marker, we also demonstrated a decrease in the microvessel density in the irradiation-treated mice, with or without AEBS, with a linear microvessel length decrease of 55.6 and $64.5 \%$ compared to the control (Fig. 7A and $B, p=0.04$ and $p=0.03$ ). Serum VEGF levels at endpoint were significantly decreased in the AEBS-treated and irradiated mice compared to the control mice (Fig. 7C).

\section{Discussion}

This study presents proof-of-concept for CA9 being a target for inhibition to increase the sensitivity of RCC cells to radiation. We elected CA9 as a target as it regulates intracellular $\mathrm{pH}$ which has been suggested to play a key protective role in irradiated cells (9). Moreover, CA9 is highly expressed on the majority of ccRCC cells, but absent in normal kidney (3). In the present study, we employed two different methods to show that CA9 confers radiation resistance in $\mathrm{RCC}$; we used knockdown of CA9 expression via transfection with specific shRNA and we inhibited the enzymatic activityof CA9 using AEBS, a sulfonamide that has previously been shown to efficiently inhibit CA9 by competition for the active site (8). We demonstrated, for the first time, that by adding AEBS to the drinking water $(50-200 \mu \mathrm{g} / \mathrm{ml})$, we achieved serum concentrations that were several magnitudes $(26.5 \mu \mathrm{M})$ higher than its $\mathrm{Ki}$ (Fig. 6D). While AEBS is relatively specific for CA9, it can also efficiently inhibit carbonic anhydrase XII (CA12) with a 10-fold lower Ki (8). Employing an agent that efficiently blocks the enzymatic activity of both CA9 and CA12 could be of therapeutic advantage, as CA12 functions similarly to 
CA9, and is also significantly expressed in RCC (10). Similar sulfonamides have been used as anti-bacterial agents before the discovery of antibiotics and are currently used as diuretics and anti-glaucoma agents due to their ability to mediate water transport and pressure in various tissues. Sulfonamides are well tolerated and are usually associated with few side effects other than potential allergic reactions (11).

The treatment of primary RCC is currently limited to surgical removal of the tumor via partial or radical nephrectomy or thermal ablation. Radiation therapy has typically been dismissed as a curative therapeutic option since RCC is generally regarded as a radiation-resistant tumor, even though normal kidney is considered radiation-sensitive. For this reason, nephropathy is a complication observed in gastrointestinal and retroperitoneal non-Hodgkin's lymphoma patients receiving abdominal radiotherapy as their primary treatment (12). Moreover, the historical landmark trial by the Copenhagen Renal Cell Cancer Study group which randomized RCC patients at high risk for recurrence post-nephrectomy to receive $50 \mathrm{~Gy}$ vs. no radiation found no survival benefit and closed prematurely due to a toxicity-related mortality rate of $20 \%$ (13). With the utilization of more accurate CT-based image-guided delivery of radiation, a retrospective study demonstrated more acceptable complication rates (14). Nevertheless, radiation treatment of RCC is currently limited to the treatment of oligometastases or inoperable disease (15).

Comparing two CA9-positive RCC cell lines (human 786-0 and murine RAG) with tumor cell lines that are considered radiation-sensitive (LNCaP) (16) and radiation-resistant (LN-18) (17), we confirmed by clonogenic survival that these RCC cells are indeed radiation resistant (Fig. 1B). Both 786-O and RAG RCC cells were significantly more resistant to radiation than glioblastoma LN-18 cells with $\mathrm{IC}_{50}$ values of 3.52, 2.29 and $1.94 \mathrm{~Gy}$, respectively.

We performed both in vitro and in vivo investigations to test the potential radiation sensitization effects of CA9 inhibition at the level of RCC cells in vitro and using human RCC tumor xenografts where the effect on tumor vascularization can be evaluated. Our study found, for the first time, that in ccRCC both the pharmacological inhibition of CA9 activity and knockdown of the expression of CA9 sensitized RCC cells to radiation in vitro (Figs. $2 \mathrm{~B}, 4 \mathrm{~A}$ and $\mathrm{B}$ ) and in vivo (Figs. 3B, $6 \mathrm{~A}$ and $\mathrm{B})$. Inhibition of CA9 activity by treatment of mice with AEBS in combination with IR had an inhibitory effect on the growth rate of the RCC xenografts and had a significantly larger effect compared with radiation alone $(\mathrm{p}=0.04$, Fig. 6A and B). Similarly, using a xenograft model of human colorectal adenocarcinoma cells, Mclntyre et al demonstrated that knockdown of CA9 reduced the growth rate of xenografts (18). Doyen et al showed that silencing of CA9 significantly increased radiation-induced cell death in another human colorectal adenocarcinoma cell line, while ectopic expression of CA9 in fibroblasts lacking CA9 expression improved survival following radiation in an acidic environment (9). Treatment of mice bearing human colon carcinoma xenografts with acetazolamide, another sulfonamide similar to AEBS, which has been demonstrated to inhibit CA9 in 786-O cells via induced apoptosis (19), also led to sensitization to radiation (20). This lends credibility to the concept that CA9 is associated with radiation tolerance by limiting a cell's ability to avoid apoptosis after irradiation.
Cells treated with AEBS or expressing shCA9 exhibited significantly reduced survival $(\mathrm{p}<0.001)$ after receiving a single dose treatment of 6 Gy compared to the fractionated radiation treatment of three doses of $2 \mathrm{~Gy}$. This reduction in survival underscores the importance of CA9 in the radiation resistance of 786-O cells and is important in the context of RCC. Whereas conventional 1.8-3.0 Gy fractions do not cause sufficient endothelial apoptosis, high-dose radiotherapy efficiently induces endothelial apoptosis via increased ceramide production and is expected to be detrimental in RCC, which is typically highly vascularized (21). While clinical evidence for the efficacy of stereotactic body radiotherapy of primary RCC is currently sparse (15), a renewed interest in this treatment option using novel CT-based image-guided radiation delivery for primary RCC has recently been expressed which awaits confirmation in the setting of prospective randomized trials (22). In our mice, IR delivered as one radiotherapy dose of 6 Gy significantly reduced the microvessel density within the tumors, alone and in combination with AEBS ( $\mathrm{p}=0.03$ and $\mathrm{p}=0.04$, respectively), whereas the mouse serum VEGF levels were decreased after IR and after inhibition of CA9 by AEBS compared with the control untreated animals ( $p=0.04$, Fig. 7).

In conclusion, our study presents experimental proof-ofconcept for the potential role of CA9 inhibition as a means to sensitize RCC to radiation. Specifically, it offers the concept of pharmacological inhibition of CA9 by sulfonamides, such as AEBS and acetazolamide, which have already been in clinical use for decades (11). While further mechanistic studies are underway, our data warrant consideration to perform phase I clinical trials.

\section{Acknowledgements}

This research was financially supported by McMaster Surgical Associates (W.C.M.D. and J.H.P.). We are grateful for the assistance by Dr Kirk Green and Sujan Fernando of the McMaster Regional Centre for Mass Spectrometry.

\section{References}

1. Buchou T, Vernet M, Blond O, Jensen HH, Pointu H, Olsen BB, Cochet $\mathrm{C}$, Issinger $\mathrm{OG}$ and Boldyreff $\mathrm{B}$ : Disruption of the regulatory beta subunit of protein kinase $\mathrm{CK} 2$ in mice leads to a cell-autonomous defect and early embryonic lethality. Mol Cell Biol 23: 908-915, 2003.

2. Mountford PJ and Temperton DH: Recommendations of the International Commission on Radiological Protection (ICRP) 1990. Eur J Nucl Med 19: 77-79, 1992.

3. Nyhan MJ, El Mashad SM, O'Donovan TR, Ahmad S, Collins C Sweeney P, Rogers E, O'Sullivan GC and McKenna SL: VHL genetic alteration in CCRCC does not determine de-regulation of HIF, CAIX, hnRNP A2/B1 and osteopontin. Cell Oncol (Dordr) 34: 225-234, 2011.

4. Grabmaier K, A de Weijert MC, Verhaegh GW, Schalken JA and Oosterwijk E: Strict regulation of CAIX(G250/MN) by HIF-1alpha in clear cell renal cell carcinoma. Oncogene 23: 5624-5631, 2004.

5. Rini BI and Small EJ: Biology and clinical development of vascular endothelial growth factor-targeted therapy in renal cell carcinoma. J Clin Oncol 23: 1028-1043, 2005.

6. Swietach P, Hulikova A, Vaughan-Jones RD and Harris AL: New insights into the physiological role of carbonic anhydrase IX in tumour $\mathrm{pH}$ regulation. Oncogene 29: 6509-6521, 2010.

7. Kleinmann N, Duivenvoorden WC, Hopmans SN, Beatty LK, Qiao S, Gallino D, Lhotak S, Daya D, Paschos A, Austin RC, et al: Underactivation of the adiponectin-adiponectin receptor 1 axis in clear cell renal cell carcinoma: Implications for progression. Clin Exp Metastasis 31: 169-183, 2014. 
8. Akurathi V, Dubois L, Lieuwes NG, Chitneni SK, Cleynhens BJ, Vullo D, Supuran CT, Verbruggen AM, Lambin P and Bormans GM: Synthesis and biological evaluation of a $99 \mathrm{mTc}-$ labelled sulfonamide conjugate for in vivo visualization of carbonic anhydrase IX expression in tumor hypoxia. Nucl Med Biol 37: 557-564, 2010.

9. Doyen J, Parks SK, Marcié S, Pouysségur J and Chiche J: Knock-down of hypoxia-induced carbonic anhydrases IX and XII radiosensitizes tumor cells by increasing intracellular acidosis. Front Oncol 2: 199, 2012.

10. Ivanov S, Liao SY, Ivanova A, Danilkovitch-Miagkova A, Tarasova N, Weirich G, Merrill MJ, Proescholdt MA, Oldfield EH, Lee J, et al: Expression of hypoxia-inducible cellsurface transmembrane carbonic anhydrases in human cancer. Am J Pathol 158: 905-919, 2001.

11. Neuman MG, Shear NH, Malkiewicz IM, Taeri M, Shapiro LE, Krivoy N, Haber J, Gomez M, Fish J, Cartotto R, et al: Immunopathogenesis of hypersensitivity syndrome reactions to sulfonamides. Transl Res 149: 243-253, 2007.

12. Kim TH, Somerville PJ and Freeman CR: Unilateral radiation nephropathy - the long-term significance. Int J Radiat Oncol Biol Phys 10: 2053-2059, 1984.

13. Kjaer M,Frederiksen PL and Engelholm SA: Postoperative radiotherapy in stage II and III renal adenocarcinoma. A randomized trial by the Copenhagen Renal Cancer Study Group. Int J Radiat Oncol Biol Phys 13: 665-672, 1987.

14. Kao GD, Malkowicz SB, Whittington R, D'Amico AV and Wein AJ: Locally advanced renal cell carcinoma: Low complication rate and efficacy of postnephrectomy radiation therapy planned with CT. Radiology 193: 725-730, 1994.

15. Svedman C, Karlsson K, Rutkowska E, Sandström P, Blomgren H, Lax I and Wersäll P: Stereotactic body radiotherapy of primary and metastatic renal lesions for patients with only one functioning kidney. Acta Oncol 47: 1578-1583, 2008.
16. Hensley HH, Hannoun-Levi JM, Hachem P, Mu Z, Stoyanova R, Khor LY, Agrawal S and Pollack A: PKA knockdown enhances cell killing in response to radiation and androgen deprivation. Int J Cancer 128: 962-973, 2011.

17. Barazzuol L, Jena R, Burnet NG, Jeynes JC, Merchant MJ, Kirkby KJ and Kirkby NF: In vitro evaluation of combined temozolomide and radiotherapy using $\mathrm{X}$ rays and high-linear energy transfer radiation for glioblastoma. Radiat Res 177: 651-662, 2012.

18. McIntyre A, Patiar S, Wigfield S, Li JL, Ledaki I, Turley H, Leek R, Snell C, Gatter K, Sly WS, et al: Carbonic anhydrase IX promotes tumor growth and necrosis in vivo and inhibition enhances anti-VEGF therapy. Clin Cancer Res 18: 3100-3111, 2012.

19. Cianchi F, Vinci MC, Supuran CT, Peruzzi B, De Giuli P, Fasolis G, Perigli G, Pastorekova S, Papucci L, Pini A, et al: Selective inhibition of carbonic anhydrase IX decreases cell proliferation and induces ceramide-mediated apoptosis in human cancer cells. J Pharmacol Exp Ther 334: 710-719, 2010.

20. Dubois L, Peeters S, Lieuwes NG, Geusens N, Thiry A, Wigfield S, Carta F, McIntyre A, Scozzafava A, Dogné JM, et al: Specific inhibition of carbonic anhydrase IX activity enhances the in vivo therapeutic effect of tumor irradiation. Radiother Oncol 99: 424-431, 2011.

21. Fuks Z and Kolesnick R: Engaging the vascular component of the tumor response. Cancer Cell 8: 89-91, 2005.

22. De Meerleer G, Khoo V, Escudier B, Joniau S, Bossi A, Ost P, Briganti A, Fonteyne V, Van Vulpen M, Lumen N, et al: Radiotherapy for renal-cell carcinoma. Lancet Oncol 15: e170-e177, 2014 